\title{
Laser-based in situ backreflection analysis of the particle size distribution in the liquid phase of bioprocesses
}

\author{
S. Junne ${ }^{1}$, C. Barilaro ${ }^{1}$, P.Neubauer ${ }^{1}$ \\ ${ }^{1}$ Chair of Bioprocess Engineering Department of Biotechnology, Technische Universität Berlin \\ Ackerstrasse 76, ACK 24, D-13355 Berlin, Germany \\ stefan.junne@tu-berlin.de
}

\begin{abstract}
Summary
A novel method for the determination of the particle and cell-size size distribution is adapted for the optimization of feedstock pre-treatment and measurement of the fluid phases of mono-cultivation and complex anaerobic digestion bioprocesses. The method relies on laserlight backreflection of particles, which are hit by a rotating focus beam. Due to the different patterns of backreflection at the edges and cores of particles, sizes of them can be determined in situ.
\end{abstract}

Keywords: Laserlight, backreflection, partice size, feedstock preparation

\section{Introduction}

Hydrolysis by complex biological cultures is an important process to brake down feedstock for the energetic use of biomass, and eventually in future, for material use as well. Hydrolysis can be operated under different process conditions. This can be the variation in temperature, which has a significant influence on the rate of decomposition, but also chemical and mechanical treatment. This includes enzymatic treatment, ultrasound, milling, and others.

Up to now, monitoring of anaerobic complex cultures and the processes operated with them is not sufficient, nor is the existing process control system capable of recording parameters that change sufficiently quickly during acidification. A good monitoring strategy for continuous processes is particularly important in order to be able to drive with short residence times and high substrate loads. Furthermore, substrate flexibility is to be increased. This increases the applicability of such a concept for closed circles and improved economic efficiency.

In case of the optimization of pre-treatment, the increase of the surface area of digestible particles play an important role to stabilize the conversion processes. However, no standard tool is applied so far in suspension at plants, and often optimization is rather based on experience than on true process values. As methane is often the final product, impacts can be seen only after weeks or months, deeper investigations are hard to perform. Therefore, this study aims to adjust and apply in situ laser-based backreflection in order to rapidly assess the particle size directly in the culture broth. Applying this approach, an increased substrate flexibility and operational efficiency is envisaged. A special focus is put on the ultrasound-based disruption of particles, which shall be monitored with laserlight-based backreflection so that a real-time in situ optimization becomes feasible in small and in industrial scale.

\section{Methods}

A laser-based reflection measurement device with single mode fiber transmission (APAS, Sequip S+E GmbH, Germany) was applied. The focus area is adjustable manually in front of a sapphire window. The variation of wavelength and laser energy for single mode light transmission allows the determination of particles in a wide range of sizes, in this case between 0.5 and $1440 \mu \mathrm{m}$. The cord length of the backreflection signal of a pulsed light is recorded and the size is determined. Light pulsation is achieved with a rotating optical disc. Laser energy and the sample chamber were adjusted to obtain substantially different signals from particles and the background, which was ignored by a tailored signal intensity threshold. 
Culture broths of continuously operated labscale anaerobic digestion fed daily with maize silage were applied as well as pure cultures, which are typically driving hydrolysis. These were a non-sporulating Bacillus subtilis cultivation and a strictly anaerobic Clostridium acetobutylicum culture. Pure cultures were applied to gain data about the bacterial size distribution before and after treatment with bead-milling, grinding and especially ultrasound. The vitality of cells was observed by plating on agar and colony counting. Ultrasound was performed either with a stand alone device or coupled with a peristaltic pump as a flow through unit (Hielscher $\mathrm{GmbH}$, Germany). The impact of enzymatic degradation on the particle-size distribution was investigated with pre-defined enzymatic solutions (Biopract $\mathrm{GmbH}$, Germany). Lab-scale digestion experiments were conducted to evaluate the impact of a changed feedstock particle size on the digestability by monitoring the carbon dioxide and methane content (BlueSens $\mathrm{GmbH}$, Germany) in the headspace of flask cultures.

\section{Results}

Results of the measurements proved the suitability of the method to finally measure the cell size distribution of pure cultures before and after treatment with ultrasound (figure 1). The same methodology was applied for complex culture broth of an anaerobic digestion process. In both cases, a cell size reduction is visible. While in pure cultures w/o any insoluble particles, cell size reduction relies on the destruction of cell agglomerates, the shift towards smaller sizes relies on mechanical destruction of non-living particles in the digestion broth mainly above a size of $6 \mu \mathrm{m}$. Hence, it is possible to distinguish between effects based on living cells and non-living particles. Furthermore, the reproducibility between samples and homogeneity of the particle and cell sizes is increased. This is an important finding, as such parameters can effect batch-to-batch reproducibility in lab scale experiments for the optimization of anaerobic digestion processes.

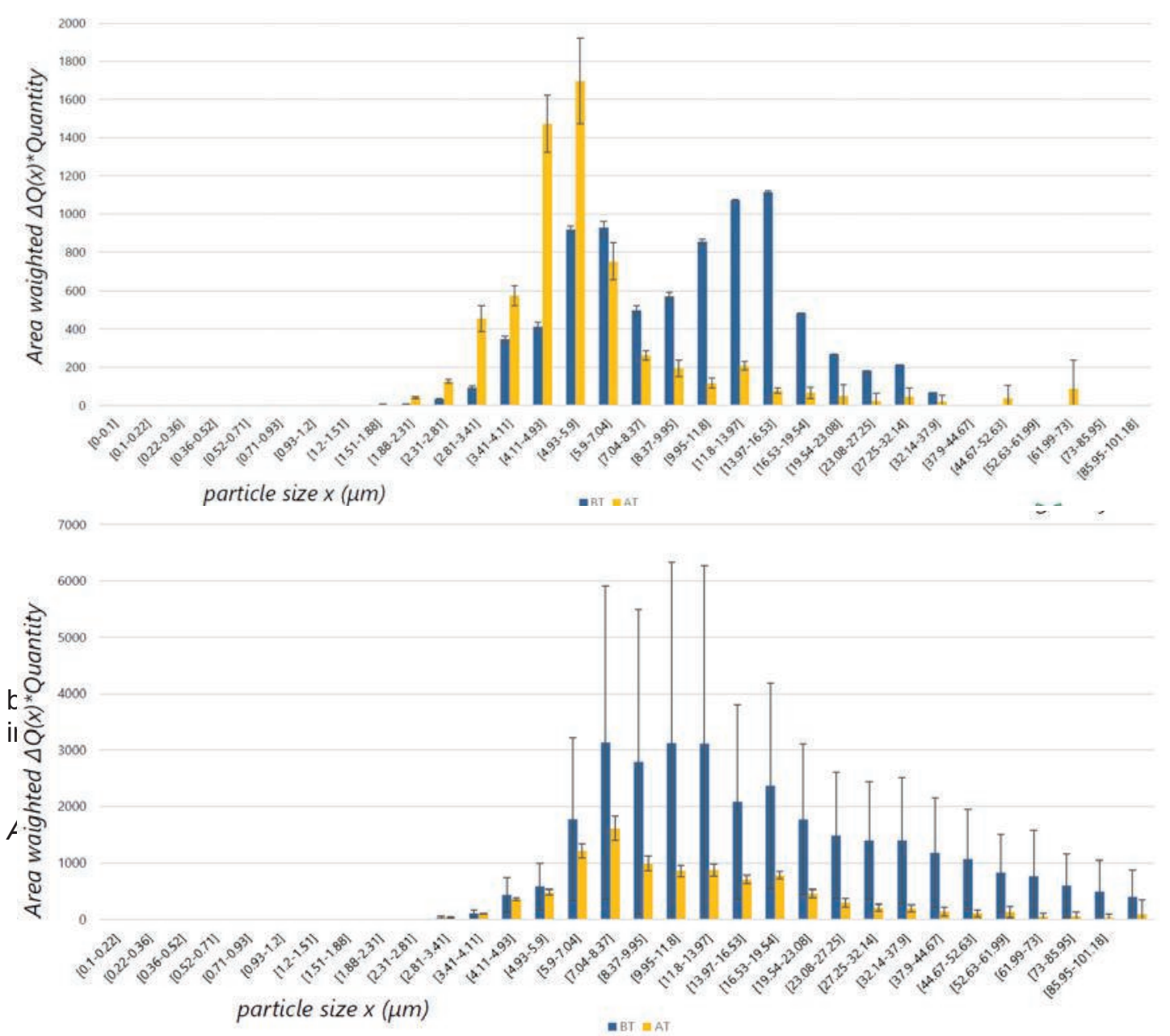

Fig. 1: Particle size distribution as measured directly in cell suspension with laserlight backreflection. Top: Size distribution of a pure culture of Bacillus subtilis before (blue) and after (yellow) treatment with ultrasound; bottom: Size distribution of digested complex culture broth before (blue) and after (yellow) treatment with ultrasound. Duration of treatment was 2 min in each case. 
As it can be seen in fig. 1, the amount of particles increases in case of a pure culture due to the case that many cells form an agglomerated particle, while in case of culture broth, a reduction of the amount of particles is seen. It shows that the solubility of particles, which were opposed to ultrasound treatment increases. This is seen at time-resolved measurements of the particle fraction: After several minutes following the ultrasound treatment, an ongoing reduction of the amount of particles is visible by the laserlight backreflection (data not shown). The application of flask scale digestion prove a faster hydrolysis and conversion to primary intermediates, like short-chain carboxylic acids.

Sonification of cell-free material like feedstock suspension of wooden material has an impact on the particle size distribution as well. Data shown in figure 2 prove the applicability of the method at high dry matter contents of up to 60 $\%$ for a broader size range. Ongoing experiments envisage the optimization of the power input of ultrasound during feedstock pretreatment. Effects are investigated with viscosity measurements as well.

\section{Conclusions}

The method allows to determine size distributions over a typical range of cell and particle densities, which are usually achieved in bioprocesses. The resolution of the technology reaches up to $0.5 \mu \mathrm{m}$, which makes it also applicable for bacterial cultivations. The results show that the method is robust and allows the rapid identification of suitable operation conditions among scales w/o the need of time-consuming activity tests or the observation of the culture performance.
The detection of agglomerates due to unfavourable stress conditions becomes feasible as well as the particle size determination in culture broth after the exposure to mechanical and physical treatment of feedstock and cells, as e.g. milling or ultrasound. Besides, the monitoring of cocultivation processes becomes possible, if the co-cultures differ in morphologic features. The applicability of the method and the potential to improve monitoring of bioprocesses is demonstrated. As usually problems occur due to the high dry matter content and high background signals at the application of many alternative technologies in anaerobic digestion, the laser-based backreflection allows to tailor feedstock and cell suspension treatment based on the particle size distribution.

\section{Acknowledgements}

The authors kindly acknowledge funding of the German Federal Ministry of Economics and Energy within the framework program "Biomass Energy Use", project "Lasersize", grant no. 03KB120.

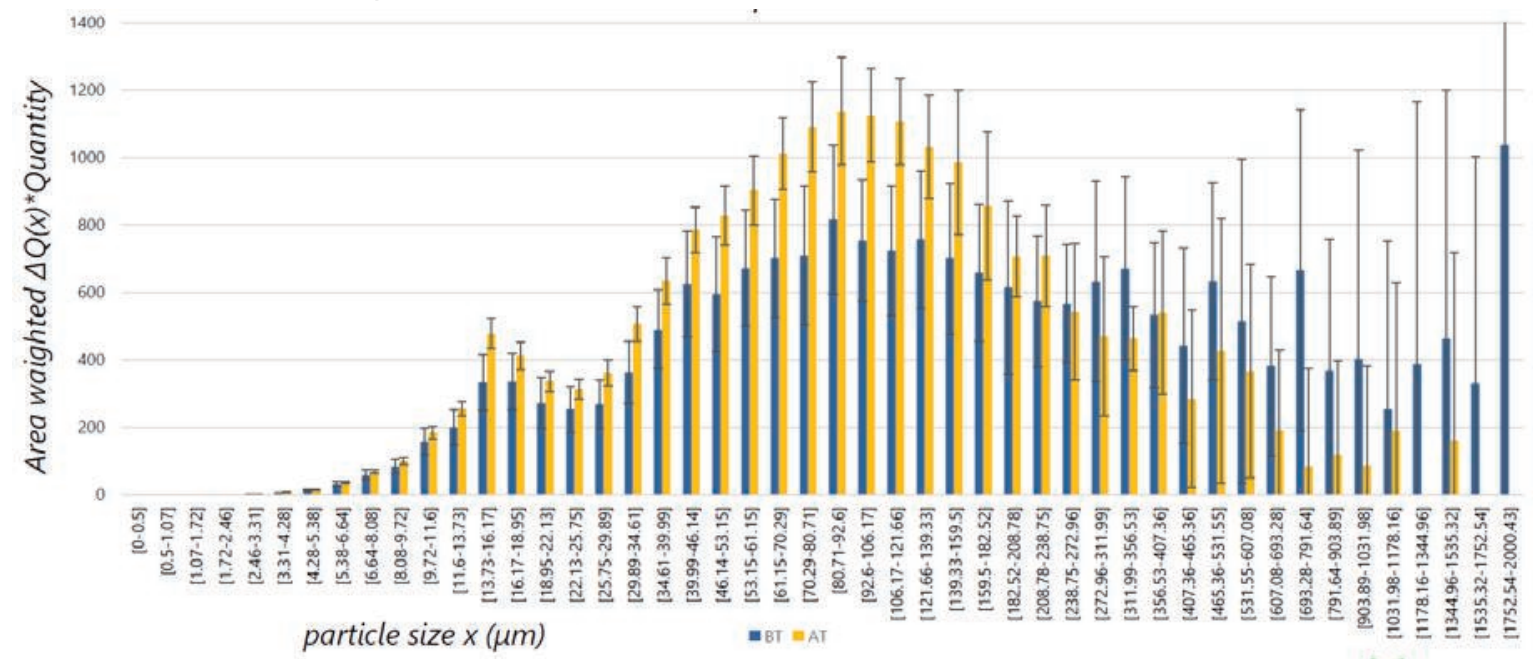

Fig. 2: Particle size distribution as measured directly in biogenic residue with laserlight backreflection and ultrasound treatment. Duration of treatment was 2 min in each case. 